\title{
Effect of the magnetic properties of the inclusions on the high-frequency dielectric response of diluted composites
}

\author{
O. Reynet, A.-L. Adenot, S. Deprot, and O. Acher* \\ Laboratoire Matériaux Magnétiques et Hyperfréquences, CEA Le Ripault, BP 16, 37260 Monts, France \\ M. Latrach \\ École Supérieure d'Électronique de l'Ouest, Département Électronique et Sciences Physiques, 4 rue Merlet de la Boulaye, \\ BP 926, 49009 Angers, France
}

(Received 13 December 2001; revised manuscript received 10 April 2002; published 12 September 2002)

\begin{abstract}
The high-frequency permittivity of composites consisting of a lattice of ferromagnetic wires is investigated. Experimental results using free space or coaxial line microwave measurements are reported. It is shown that the dielectric response is strongly dependent on the magnetic properties of the wires. Negative real permittivity is observed over a wide frequency range for wires with circumferential magnetization, while a resonant behavior is observed on wires with an axially magnetized core. In addition, it is shown that a moderate external field can induce large changes in the dielectric response. We prove that the underlying physics of these composites made of oriented magnetic wires is basically the same as the giant magnetoimpedance (GMI) effect. A model based on GMI equations is proposed which predicts this unusual dielectric phenomenon.
\end{abstract}

DOI: 10.1103/PhysRevB.66.094412

PACS number(s): 75.80.+q, 77.84.Lf

\section{INTRODUCTION}

The design of composite materials with attractive and new electromagnetic properties has been an important issue for years. ${ }^{1-3}$ A recent success that has focused great attention is the experimental observation of materials with negative index at microwave frequencies ${ }^{4}$ and the demonstration of the new possibilities opened by this class of materials. ${ }^{5,6}$ These achievements have been possible by a prior understanding of the way to design negative permeability using metallic inclusions ${ }^{7}$ and negative permittivity from a lattice of conducting wires. Other routes to negative index material have been reported ${ }^{8}$ also using nonmagnetic conducting inclusions. In the frame of a more general search to engineer the spectral response of materials, composites made of highly diluted finite-length nonmagnetic conductive fibers have been shown to engineer the permittivity. ${ }^{9,10}$ In particular, it has been established both theoretically ${ }^{10}$ and experimentally ${ }^{9}$ that the permittivity of diluted fiber-based materials is strongly influenced by the skin effect. However, these studies were restricted to the case of nonmagnetic fibers. The use of conductive nonmagnetic inclusions to obtain permeability levels different from unity is also known. ${ }^{11}$ In contrast, to our knowledge, the use of magnetic materials to engineer the dielectric response has never been considered. One purpose of this paper is to demonstrate this possibility. For this purpose, lattices of thin continuous wires are manufactured and studied in the microwave range, and the spectral response is investigated for different magnetic properties of the wires.

A now well-established class of high-frequency materials is photonic band gap materials. ${ }^{2} \mathrm{~A}$ recent trend ${ }^{12}$ is to achieve the adjustability of these materials. This is usually done using an electric-field-driven material or electronic component. Another purpose of this paper is to demonstrate a large adjustability of the dielectric response through an external magnetic field of the class of composites considered here. This contrasts with most results on tunable materials, where it is common to tune the permeability $\mu$ through an external magnetic field $\mathbf{H}$ (Refs. 13 and 14) and the permittivity $\varepsilon$ through an electric field $\mathbf{E}$, but not $\varepsilon$ through $\mathbf{H}$ as demonstrated here. We propose a model to account for the behavior observed in these composites made of oriented magnetic wires. We prove that the underlying physics has strong connection with that of the giant magnetoimpedance (GMI) effect. ${ }^{15,16}$ As a consequence, composites with engineered or tunable dielectric response made from diluted magnetic inclusions are at hand, using the strong theoretical and experimental background in GMI on magnetic wires and ribbons.

\section{EXPERIMENTAL DETAILS AND RESULTS}

\section{A. Lattice of ferromagnetic wires}

Amorphous glass-covered microwires (AGCW's) have been produced with our facility. ${ }^{17}$ These wires consist of a $10-\mu \mathrm{m}$ (CoFeSiB) amorphous core, covered by a $5-\mu \mathrm{m}$-thick glass shell. The static magnetic properties of such microwires have been throughly investigated, ${ }^{18}$ and it is well known that a key feature that determines the magnetization distribution within the wire is the sign and magnitude of the magnetostriction coefficient. ${ }^{19}$ Large internal strains created by the outer glass shell on the magnetic core can lead to a dominant contribution of magnetoelastic effects on the anisotropy and domain structure. Three compositions were manufactured with different $\mathrm{Fe} /(\mathrm{Co}+\mathrm{Fe})$ ratios, in order to yield small positive, small negative, and large positive magnetostriction coefficient $\lambda_{s}$. The diameter are similar, and the electrical resistivity is close to $130 \mu \Omega \mathrm{cm}$ for all the wires. The characteristics of the wires are summarized in Table I.

Hysteresis measurements are reported in Fig. 1. The magnetization is mainly along the wire axis for W1 and W3, which is consistent with the positive magnetostriction coefficients for these alloys. The hysteresis loop for W2 is consistent with a large shell with circumferential anisotropy. 
TABLE I. Ferromagnetic wire properties.

\begin{tabular}{lcccc}
\hline \hline Wires & $\begin{array}{c}\text { Metallic core } \\
\text { diameter }(\mu \mathrm{m})\end{array}$ & $\begin{array}{c}\text { Total } \\
\text { diameter }(\mu \mathrm{m})\end{array}$ & $\begin{array}{c}4 \pi M_{s} \\
(\mathrm{kG})\end{array}$ & $\lambda_{s}$ \\
\hline $\mathrm{W} 1$ & 9.5 & 15 & 7.5 & Small positive \\
$\mathrm{W} 2$ & 10.5 & 14 & 6 & Small negative \\
W3 & 9 & 16 & 18 & Large positive \\
\hline
\end{tabular}

Composite materials are manufactured by placing series of parallel wires on $300 \mathrm{~mm} \times 300 \mathrm{~mm}$ polystyren foam tiles, with equal in-plane spacing $d$ between the wires (see Fig. 2). The foam tiles are $3.0 \mathrm{~mm}$ thick and can be stacked in order to obtain a two-dimensional (2D) array of parallel wires. The permittivity of the foam is within $5 \%$ of unity. The permeability and permittivity of these materials in the 2-18 GHz range are determined from reflection-transmission measurements in free space. The experimental setup ${ }^{20}$ is comprised of two microwave horns with Teflon lenses. The illuminated area is a disk with a characteristic diameter of 10-20 cm, depending on the frequency. A $25-\mathrm{cm}$-diam circular mask made of radar absorbing material suppresses any parasitic illumination of the edges of the plate under investigation. The experiment is therefore essentially representative of the response of infinitely long wires, as in similar experiments, ${ }^{21}$ though the distribution of the electric field is not uniform on the sample. Although parallel wire grids have already been studied, ${ }^{22,23}$ no lattice of ferromagnetic wires has been investigated to our knowledge yet. For the polarization with the $\mathbf{E}$ field normal to the wires, $\varepsilon$ and $\mu$ are found to be close to unity, within the precision measurement, which is about 5\%. This is because the interaction of $\mathbf{E}$ with the wires is negligible, and the volume fraction of permeable material is too small to yield a significant $\mu$. The polarization of interest is indeed for $\mathbf{E}$ parallel to the wires.

In this polarization, permeability is found to be close to unity and the quantity of interest will be the permittivity of the composites all through the paper. The results for a com-

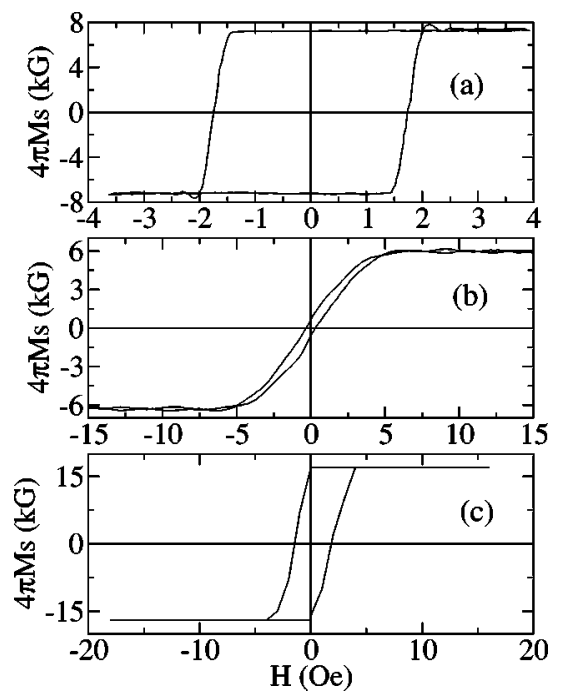

FIG. 1. Hysteresis curves of the microwires: (a) W1, (b) W2, and (c) W3.

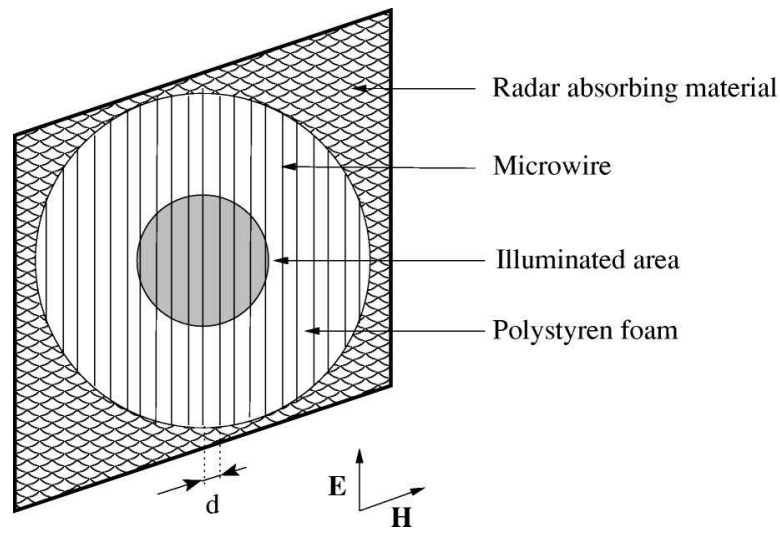

FIG. 2. 2D-wire lattice on the polystyren foam and polarization of interest.

posite made of five tiles manufactured from wire W1 with 10-mm in-plane spacing are reported in Fig. 3. As the manufacture of such tiles is very long and delicate, measurements were performed for a decreasing number of tiles in the stack, assuming a composite thickness of $3 \mathrm{~mm}$ times the number of tiles. Indeed, the permittivity was found nearly independent of the number of tiles. For that reason, all subsequent measurements were performed on one-tile-thick composites. It is known that interface effects are not negligible in the general case ${ }^{24}$ and the effective properties of a composite should be defined only for a minimum thickness. But in the present case, it seems that measurements performed on one tile yield the proper order of magnitude and line shape.

The effect of decreasing the in-plane spacing $d$ from 10 $\mathrm{mm}$ down to $5 \mathrm{~mm}$ is reported in Fig. 4. It appears that the dielectric susceptibility $\varepsilon-1$ doubles when the surface wire fraction doubles. The dielectric response exhibits a resonance below $2 \mathrm{GHz}$. Negative permittivity is achieved in the $4.3-12 \mathrm{GHz}$ range. The permittivity rises to values close to unity at higher frequencies.

The spectral response of a composite tile made of wire W2 with in-plane spacing of $10 \mathrm{~mm}$ is presented in Fig. 5. The permittivity of a composite tile made with wire W3 and in-plane spacing $d=10 \mathrm{~mm}$ is represented in Fig. 6. The

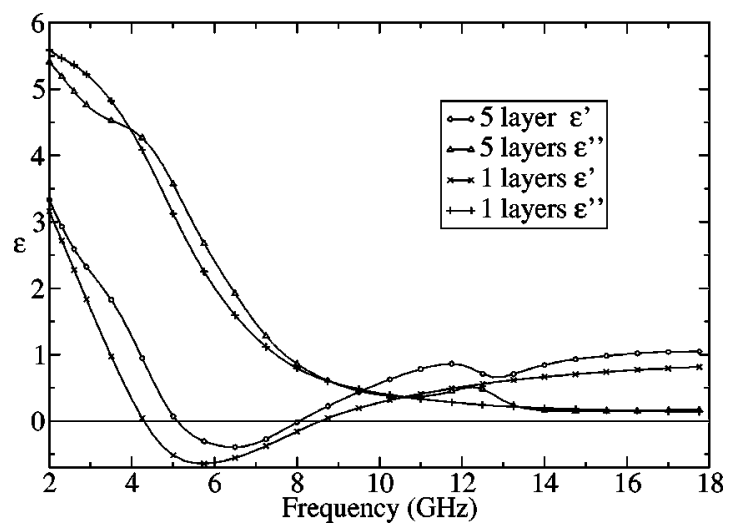

FIG. 3. Real and imaginary parts of the permittivity of one and five layers of $\mathrm{W} 1$ composite vs frequency in the $2-18 \mathrm{GHz}$ range measured in free space. Microwave $\mathbf{E}$ field is parallel to the wires. 


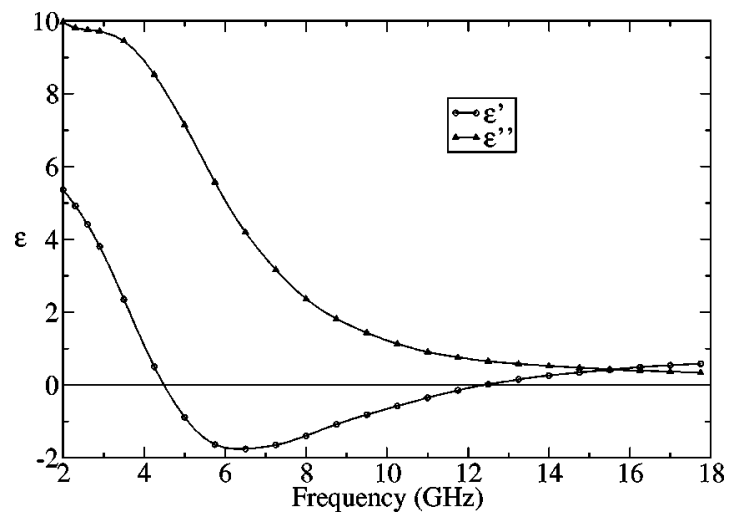

FIG. 4. Real and imaginary parts of the permittivity of a onelayered sample of 5-mm-spaced W1 composite vs frequency in the $2-18 \mathrm{GHz}$ range measured in free space. Microwave $\mathbf{E}$ field is parallel to the wires.

response differs from the two previous materials. For comparison purposes, a composite tile made with $10-\mu \mathrm{m}$-diam copper wires and in-plane spacing $d=10 \mathrm{~mm}$ has been manufractured, and the permittivity is reported in Fig. 7.

As all the wires have nearly identical geometrical and electrical characteristics, the difference between the composites in Figs. 3, 5, and 6 should be attributed to the differences in their magnetic properties. All these wires have soft magnetic properties, and they exhibit large magnetization changes for small external applied fields (see Fig. 1). As a consequence, it is natural to investigate the response of these composites under magnetic field and to search for the adjustability of $\varepsilon$ through a magnetic field. However, measurements under magnetic field are not easily done on $300 \mathrm{~mm}$ $\times 300 \mathrm{~mm}$ tiles with a free-space microwave measurement apparatus and should rather be performed in a waveguiding structure.

\section{B. Tunable permittivity}

A second type of microwave measurements was made using an APC-7 coaxial line. A 9-mm-long piece of wire W1 is placed on a sample holder as sketched in Fig. 8. Small grooves were machined in the internal and external conduc-

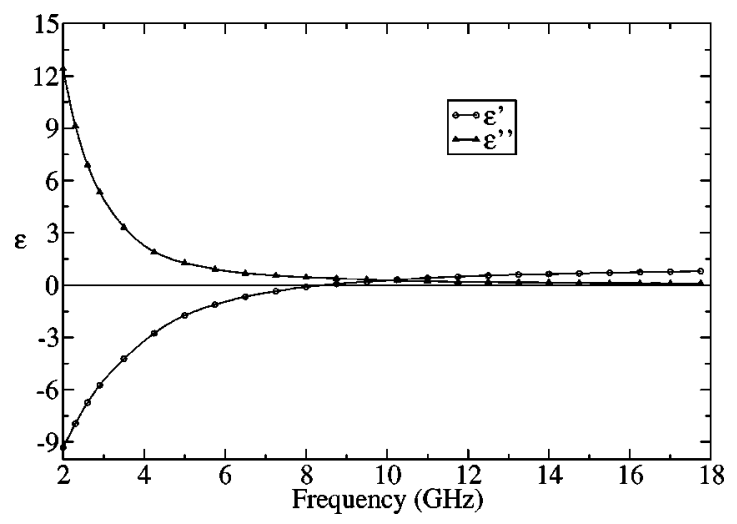

FIG. 5. Real and imaginary parts of the permittivity of a onelayered sample W2 composite vs frequency in the $2-18 \mathrm{GHz}$ range measured in free space. Microwave $\mathbf{E}$ field is parallel to the wires.

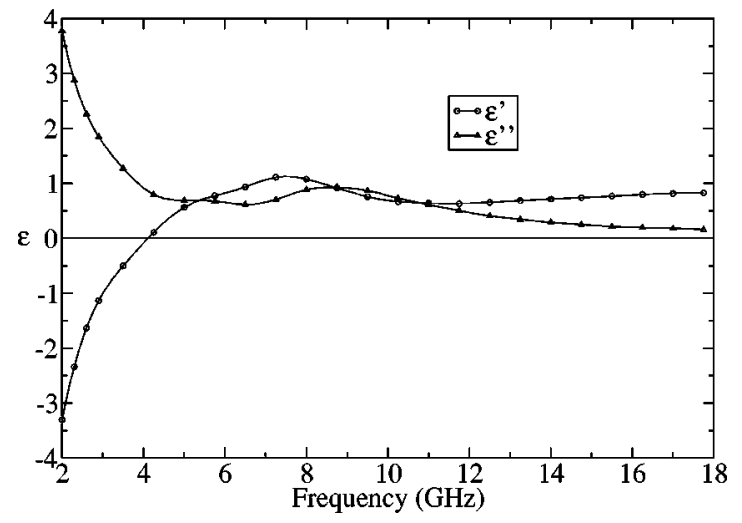

FIG. 6. Real and imaginary parts of the permittivity of a onelayered sample $\mathrm{W} 3$ composite vs frequency in the $2-18 \mathrm{GHz}$ range measured in free space. Microwave $\mathbf{E}$ field is parallel to the wires.

tors, in order to place the wire, and filled with indium. This prevents the wire from being crushed when it is inserted in the coaxial line. The wire is parallel to the microwave $\mathbf{E}$ field. There is no electrical contact between the ferromagnetic core of the wire and the inner or the outer conductor of the coaxial line, due to the glass cover. As the wire extends from the inner conductor to the outer conductor, the measured properties are representative of free-space measurements on samples with an infinite sequence of short chained dipoles separated by a very small distance that correspond to the glass thickness, with a horizontal spacing $d$ that is of the order of half the circumference of either the inner diameter or the outer diameter of the line.

A static Ho field parallel to the wire can be applied, using Helmholz coils. The reflection and transmission coefficients through the samples are measured in the range from $1 \mathrm{MHz}$ to $6 \mathrm{GHz}$, using a network analyzer. The sample is then described as the stacking of a composite material containing a wire with an arbitrary thickness $e$ small compared to the wavelength and of a Plexiglass ring with known permittivity. The $\varepsilon$ and $\mu$ of the composite is determined from the $R / T$ coefficients using the Fresnel equations. The permeability is found to be close to unity within the 5\% uncertainty mea-

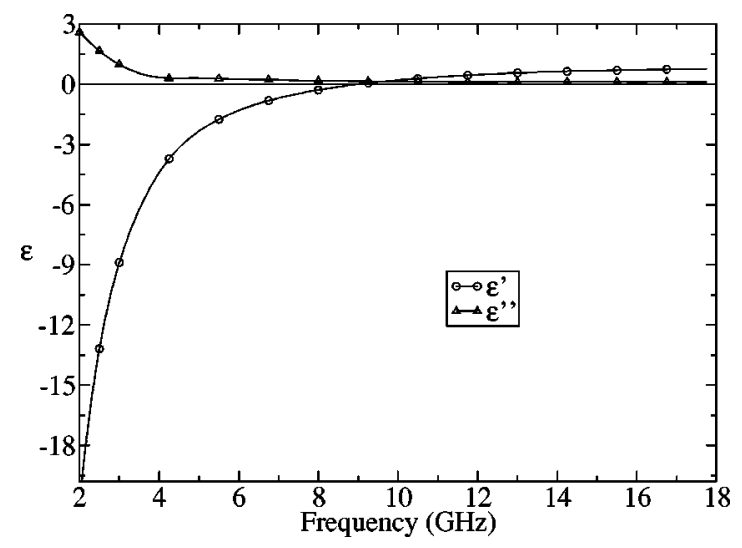

FIG. 7. Real and imaginary parts of the permittivity of a onelayered sample $\mathrm{Cu}$-wire composite vs frequency in the $2-18 \mathrm{GHz}$ range measured in free space. Microwave $\mathbf{E}$ field is parallel to the wires. The metallic core diameter is $10 \mu \mathrm{m}$. 


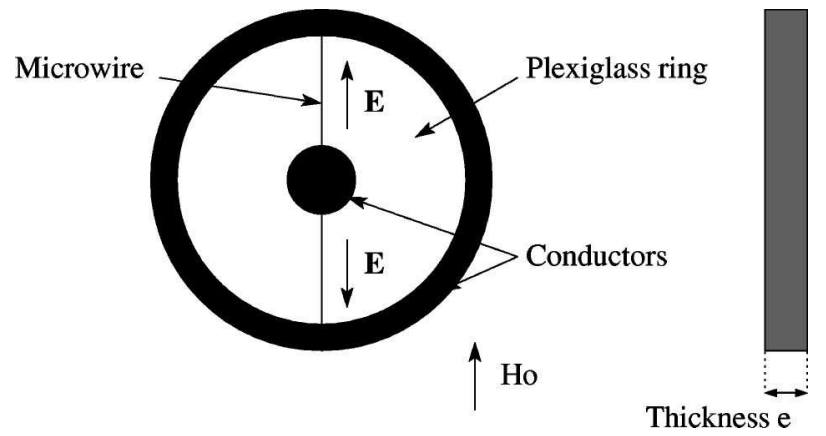

FIG. 8. APC7 sample with microwire inclusion in a Plexiglass ring.

surements, as expected. A thickness cannot be attributed to these planar composites. Nevertheless, the thickness permittivity product $e \varepsilon$ is found to be independent of $e$ (see Sec. III). As a consequence, vertical axes are labeled with the product $e \varepsilon$.

The permittivity obtained for wire $\mathrm{W} 1$ is represented in Fig. 9 for static values of the field ranging from 0 Oe to 130 Oe. The results were checked to be reproducible and independent of the magnetic history. The influence of the Plexiglass, which has a relative permittivity of 2.75 , has been suppressed. The permittivity is clearly changing with static magnetic field. The permittivity exhibits a resonant behavior, with some similarities with free-space measurements on the W1-based composite of Figs. 3 and 4, but at a lower frequency.

A similar experiment (see Fig. 10) was made with the wire W2, which has a negative magnetostriction coefficient. Slowly increasing Ho really modifies the permittivity of the composite. Negative permittivity of the composite can be achieved in a variable range of frequency, depending of the applied static magnetic field.
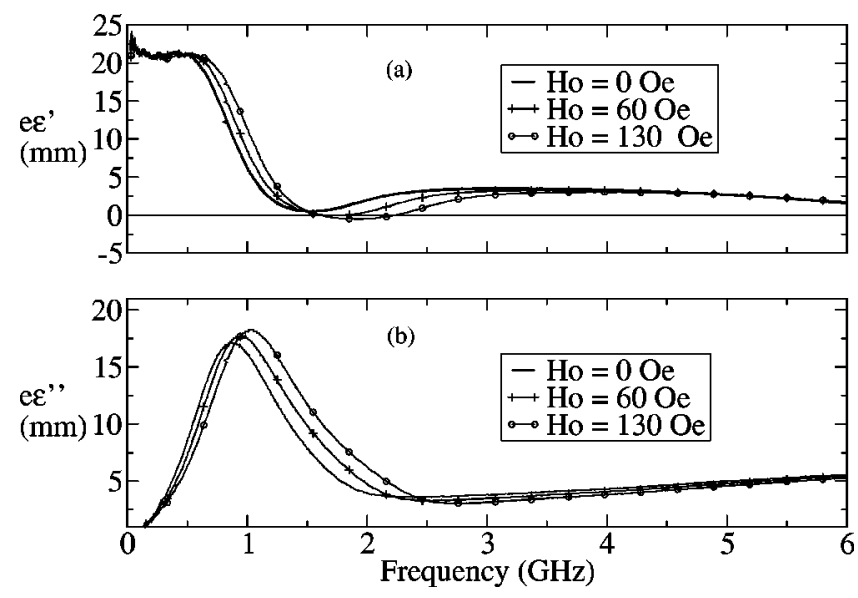

FIG. 9. Permittivity by thickness product of W1 composite vs frequency in the range from $1 \mathrm{MHz}$ to $6 \mathrm{GHz}$ measured in the coaxial line, for different values of a static magnetic field. Microwave $\mathbf{E}$ and static $\mathbf{H o}$ field are parallel to the wire: (a) real part, (b) imaginary part.
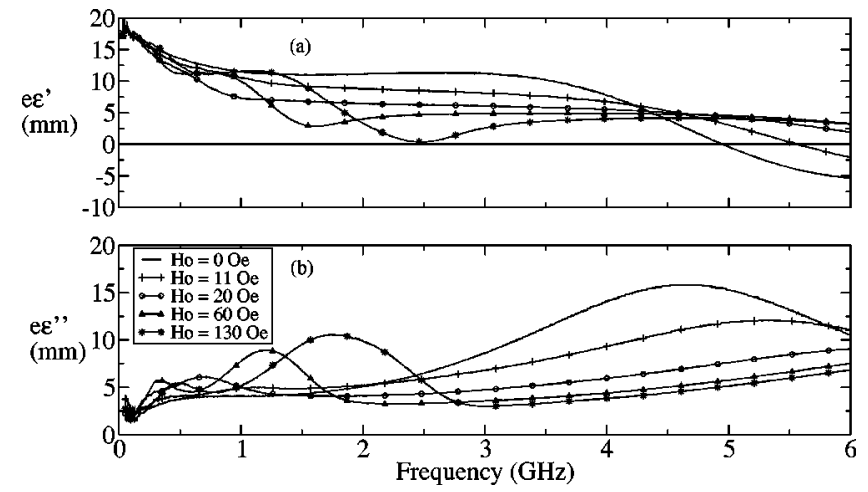

FIG. 10. Permittivity by thickness product of W2 composite vs frequency in the range from $1 \mathrm{MHz}$ to $6 \mathrm{GHz}$ measured in the coaxial line, for different values of a static magnetic field. Microwave $\mathbf{E}$ and static Ho field are parallel to the wire: (a) real part, (b) imaginary part.

\section{THEORETICAL APPROACH}

\section{A. Effective permittivity}

Let us consider a composite made of a 2D lattice of infinitely long parallel wires in a matrix with relative permittivity $\varepsilon_{m}$ and relative permeability $\mu=1-j 0$. Let us consider the properties of this composite for an incident propagation vector and microwave $\mathbf{H}$ field perpendicular to the wires and incoming $\mathbf{E}$ field parallel to the wires. Let us suppose in addition that the periods $d$ (spacing) and $e$ (thickness) are much smaller than the wavelength and that the filling fraction of the wire, denoted $f$, is much smaller than unity. Effective medium theories are widely used to describe the microwave properties of composite materials, ${ }^{10}$ and the aim is to determine the effective quantities $\mu_{e f f}$ and $\varepsilon_{e f f}$ of such a composite. Different definitions of the effective permeability and permittivity are found in the literature, ${ }^{7,25}$ and it has been shown that the correct definition yielding effective quantities that are consistent with the conventional expression of the reflection and transmission coefficients should be $\mathrm{be}^{26}$

$$
\begin{gathered}
\varepsilon_{0} \varepsilon_{e f f}=\frac{\langle\mathbf{D}\rangle_{\pi}}{\langle\mathbf{E}\rangle_{V}}, \\
\mu_{0} \mu_{e f f}=\frac{\langle\mathbf{B}\rangle_{V}}{\langle\mathbf{H}\rangle_{\pi}},
\end{gathered}
$$

where \langle\rangle$_{V}$ corresponds to the average over the volume of the periodical cell and \langle\rangle$_{\pi}$ corresponds to the average over a plane $\pi$ normal to the incoming $\mathbf{E}$ vector. This result has been established with the hypothesis that no conducting element is present in the $\pi$ plane. This condition is not met in the present case, but it is believed that for the very low volume fraction of metal considered here, this result is still valid. Indeed, as the system is invariant for a translation along the wire axis, the former definition reduces in that case to

$$
\varepsilon_{0} \varepsilon_{e f f}=\frac{\langle\mathbf{D}\rangle_{V}}{\langle\mathbf{E}\rangle_{V}},
$$




$$
\mu_{0} \mu_{e f f}=\frac{\langle\mathbf{B}\rangle_{V}}{\langle\mathbf{H}\rangle_{V}}
$$

In the matrix, the $\mathbf{E}$ and $\mathbf{D}$ fields are nearly constant within a periodical cell, and $\mathbf{D}=\varepsilon_{0} \varepsilon_{m} \mathbf{E}$. At the surface of the wire, the component $\mathbf{E}_{\text {ext }}$ of the $\mathbf{E}$ field parallel to the wire is continuous. Let us introduce the impedance $Z$ of an isolated piece of wire of length $l_{\text {wire }}$, following the conventional approach for the GMI effect. The present study is restricted to the case where the impedance tensor of the wire is diagonal. Then we have

$$
U=Z I=Z \iint_{\Sigma_{\text {wire }}} \mathbf{J} d \mathbf{S},
$$

where $I$ is the current flowing in the wire and $\mathbf{J}$ the current density.

The wire is a conductor with dc resistance $R_{d c}$, conductivity $\sigma_{0}$, and section $\Sigma_{\text {wire }}$ :

$$
\begin{gathered}
\mathbf{J}=j \omega \mathbf{D} \\
\mathbf{E}_{\text {ext }} l=j \omega \frac{Z}{R_{d c}} R_{d c} \Sigma_{\text {wire }}\langle\mathbf{D}\rangle_{\text {wire }},
\end{gathered}
$$

which is also written as

$$
\langle\mathbf{D}\rangle_{\text {wire }}=\frac{\sigma_{0} \mathbf{E}_{e x t}}{j \omega \frac{Z}{R_{d c}}}
$$

An expression of $\mathbf{E}_{e x t}$ as a function of $\langle\mathbf{E}\rangle_{V}$ is necessary to derive $\varepsilon_{e f f}$. The incident electric field creates a current in the microwire which generates a magnetic field. ${ }^{27}$ In a cylindrical coordinate system, this field is equal to

$$
\vec{H}_{w}(r)=\frac{I_{w}}{2 \pi r} \vec{e}_{\theta},
$$

where the current is given by

$$
I_{w}=\frac{R_{d c}}{Z} \sigma_{0} \Sigma_{w i r e} \mathbf{E}_{e x t} .
$$

Let us apply the Maxwell-Faraday integral law to the contour $\Gamma$, as sketched in Fig. 11:

$$
\oint_{\Gamma} \vec{E} \vec{d} l=-j \mu_{0} \omega \iint_{S} \vec{H} \vec{d} S
$$

where $S$ is the surface outlined by $\Gamma$.

It is found that

$$
E(r)=\mathbf{E}_{e x t}+j \mu_{0} \omega \iint_{S} \vec{H} \vec{d} S .
$$

Using Eq. (10), the average of this electric field over a periodic cell volume is

$$
\langle\mathbf{E}\rangle_{V}=\mathbf{E}_{\text {ext }}\left(1+j \mu_{0} \sigma_{0} \omega f \frac{R_{d c}}{2 \pi Z} \mathcal{A}\right),
$$

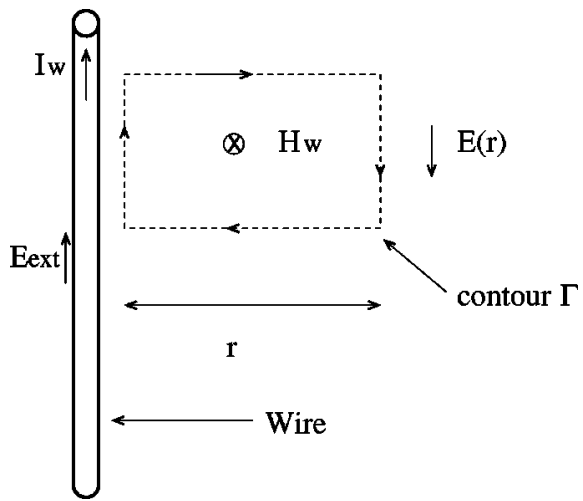

FIG. 11. Description of the contour used for the calculation of $\langle\mathbf{E}\rangle_{V}$ as a function of $\mathbf{E}_{e x t}$.

where $\mathcal{A}$ is a surface that only depends on the topology of the lattice and $f$ the volume fraction of wire defined by

$$
f=\frac{\Sigma_{\text {wire }}}{\Sigma_{\text {cell }}}=\frac{\pi a^{2}}{e d},
$$

where $\Sigma_{c e l l}$ is the area of a periodic cell, $a$ the wire radius, $d$ the spacing, and $e$ the thickness of the periodic structure. $\mathcal{A}$ can be approximated ${ }^{21}$ by taking into account the contribution of one wire only per cell and by supposing that the cell is a square one with a period $d$ between the wires such as $d \gg a$ :

$$
\mathcal{A}=d^{2} \ln (d / a)
$$

The effective permittivity for a $2 \mathrm{D}$ lattice of microwires can be deduced from relations (3), (8), and (13):

$$
\varepsilon_{e f f}=(1-f) \varepsilon_{m}+f \frac{\sigma_{0}}{j \omega \varepsilon_{0} \frac{Z}{R_{d c}}\left(1+j \omega \frac{\mu_{0} \sigma_{0} f}{2 \pi} \frac{R_{d c}}{Z} \mathcal{A}\right)}
$$

Let us introduce $c_{0}$, the speed of light, and $Z_{0}=120 \pi$, the impedance of vacuum. If we note

$$
\omega_{0}=2 \pi c_{0} \frac{Z}{Z_{0} l_{\text {wire }}} \frac{\Sigma_{\text {cell }}}{\mathcal{A}},
$$

where $Z / l_{\text {wire }}$ is the impedance per unit of length of the wire, it becomes

$$
\varepsilon_{e f f}=(1-f) \varepsilon_{m}+f \frac{\sigma_{0}}{j \omega \varepsilon_{0} \frac{Z}{R_{d c}}\left(1+j \frac{\omega}{\omega_{0}}\right)} .
$$

Here $Z / R_{d c}$ is the quantity of interest in the field of longitudinal GMI. Its dependence with frequency, external field, and bias current as a function of the characteristics of the magnetic wire has been studied extensively both experimentally and theoretically. ${ }^{28,29}$ For a wire with uniform electric and magnetic properties, the expression $Z / R_{d c}$ is given as

$$
\frac{Z}{R_{d c}}=\frac{k a}{2} \frac{J_{0}(k a)}{J_{1}(k a)},
$$


where $k$, the wave vector, is equal to

$$
k=\sqrt{-j \omega \mu_{0} \mu_{\theta} \sigma_{0}},
$$

where $\mu_{\theta}$ is the circumferential permeability of the wire.

\section{B. Comparison with other analytical models}

Equation (18) shows that the behavior of $\varepsilon_{e f f}$ is conditioned by $\omega / \omega_{0}$. Here $\omega_{0}$ is the product of a geometrical parameter $\Sigma_{\text {cell }} / \mathcal{A}$ by a factor $2 \pi c_{0} Z /\left(Z_{0} l_{\text {wire }}\right)$, which is characteristic of the electrical microwave behavior of the wire. Using the approximation (15) of $\mathcal{A}$, it yields

$$
\omega_{0}=\frac{Z}{Z_{0}} \frac{2 \pi c_{0}}{l_{\text {wire }} \ln (d / a)} .
$$

When $\omega \ll \omega_{0}$ and for $f \ll 1$, the inhomogeneities of the electric field outside the wires can be neglected. $\langle E\rangle_{V}$ $\approx E_{\text {ext }}$, as can be seen from Eq. (13). The permittivity is clearly associated with the GMI effect. Expression (16) reduces to

$$
\varepsilon_{e f f} \approx \varepsilon_{m}+f \frac{\sigma_{0}}{j \omega \varepsilon_{0} \frac{Z}{R_{d c}}} .
$$

Combining Eqs. (14) and (22) leads to the expression of the product $e \varepsilon$, which is independent of $e$ :

$$
\left(\varepsilon_{e f f}-\varepsilon_{m}\right) e \approx \frac{\pi a^{2} \sigma_{0}}{j \omega d \varepsilon_{0} \frac{Z}{R_{d c}}} .
$$

At this stage, it is interesting to compare the expression of the permittivity given by Eqs. (22), (19), and (20) to that previsouly established ${ }^{10}$ for a dispersion of nonmagnetic wires with finite length. From Eq. (44) in Ref. 10, which is based on effective medium theory, it can be shown that in the approximation of wires with a length much larger than the wavelength, the dielectric function is given by

$$
\varepsilon_{e f f} \approx \varepsilon_{m}+\frac{2}{9} \frac{f}{1-f} \frac{\sigma_{0}}{j \omega \varepsilon_{0} \frac{Z}{R_{d c}}} .
$$

Equation (24) is similar to Eq. (22) with $\mu_{\theta}=1$, provided the volume fraction $f$ is replaced by $\frac{2}{9} f /(1-f)$. As $(1-f)$ is close to unity, the only difference lies in the factor $2 / 9$ that can be attributed to the difference between the two topologies. Reference 10 considers that "sticks are supposed to be randomly distributed and oriented," while we are considering infinitely long aligned wires. This accounts for a factor $1 / 3$ in Eq. (24). This remaining 2/3 factor in Eq. (24) comes from the choice of a spherical shape to derive the polarizability of the dielectric region in the frame of effective medium theory (EMT) [see Eq. (43) in Ref. 10]. Thus, the EMT approach leads to a very similar expression to that obtained with our approach when $\omega \ll \omega_{0}$.

Now, when $\omega \gg \omega_{0}$, expression (18) reduces to

$$
\varepsilon_{e f f}=(1-f) \varepsilon_{m}-\frac{2 \pi c_{0}^{2}}{\omega^{2} \mathcal{A}} .
$$

This equation is commonly written as

$$
\varepsilon_{e f f}=(1-f) \varepsilon_{m}-\frac{\omega_{p}^{2}}{\omega^{2}},
$$

where $\omega_{p}^{2}=2 \pi c_{0}^{2} / \mathcal{A}$ is known as the plasma frequency. Using Eq. (15) for $\mathcal{A}$, we have

$$
\omega_{p}^{2} \approx \frac{2 \pi c_{0}^{2}}{d^{2} \ln (d / a)} .
$$

This expression of the permittivity only depends on the geometry, and it is in agreement with previous works ${ }^{21,27}$ on lattices of nonmagnetic wires with a strong skin effect [see, for example, Eq. (7.69) in Ref. 27]. It is remarkable to note that in this frequency regime, the addition of magnetic properties to the wires does not affect the composite permittivity. In addition, the electric field outside the wire is very inhomogeneous, as can be seen from Eq. (13). In any effective medium approach, the description of the composite through an effective permittivity is limited to the case where $2 \pi c_{0} / d \gg \omega$. Using the approximation (15) of $\mathcal{A}$, the range of validity of Eq. (26) is $2 \pi c_{0} / d \gg \omega / \omega_{0} \gg 1$. This range of validity exists provided that

$$
\frac{Z_{0}}{Z} \frac{l_{\text {wire }}}{d} \ln (d / a) \gg 1
$$

\section{TUNABLE PERMITTIVITY MECHANISM}

\section{A. Structures of magnetization in the microwires}

The permeability $\mu_{\theta}$ in this expression is the permeability of the wire for a circumferential $\mathbf{H}$ field excitation created by the current flowing through the wire. It should not be confused with the parallel permeability ${ }^{30,31}$ or the permittivity normal to the wire axis. At the $\mathrm{GHz}$ frequencies considered here, the only contribution to $\mu_{\theta}$ is the gyromagnetic permeability, as opposed to the domaine wall permeability. The available understanding of the magnetic domain structure in AGCW's allows a first evaluation of the $\mu_{\theta}$. The domain structure in AGCW's is governed by the built-in stress and the magnetostriction coefficient of the amorphous alloy. ${ }^{16}$ Indeed, the glass cover creates a large tensile stress in the metallic part, and as a consequence the magnetic anisotropy essentially results from the magnetoelastic contribution. For negative magnetostriction alloys (W2), this results in a shell with a circumferential "bamboolike" domain structure [Fig. 12(a)].

The structure of the core is still under debate. ${ }^{32}$ For positive magnetostriction alloys (W1 and W3), the core is axially magnetized and a thin outer shell has a more complex structure [see Fig. 12(b)]. The microwave response is governed by the Landau-Gilbert equation ${ }^{33,34}$ 


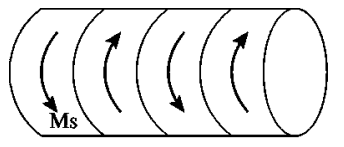

(a)

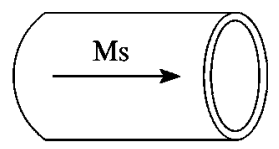

(b)
FIG. 12. Sketch of domain structure for Co-based wires: (a) with negative magnetostriction, (b) with positive magnetostriction.

$$
\frac{d \mathbf{m}}{d t}=-\gamma \mathbf{m} \times \mathbf{H}+\frac{\alpha}{m} \mathbf{m} \times(\mathbf{m} \times \mathbf{H}),
$$

where $\mathbf{m}$ is the magnetization, $\mathbf{H}$ the total magnetic field, $\gamma$ the gyromagnetic ratio, and $\alpha$ the damping parameter.

\section{B. Analytical model versus experiment}

If the inner core structure of negative magnetostriction wire W2 is neglected, it is clear from Fig. 12(a) that the microwave magnetic field created by a current flowing through the wire is parallel to the magnetization. Equation (29) then shows that $d \mathbf{m} / d t=0$, and as a consequence the circumferential permeability $\mu_{\theta}$ is unity. In this case, the wire behaves like a conducting nonmagnetic wire. Using Eq. (18), good agreement is achieved with the free-space measurements on the W2-based composite as shown in Fig. 13. The values of the fit are reported in Table II. It can be seen in

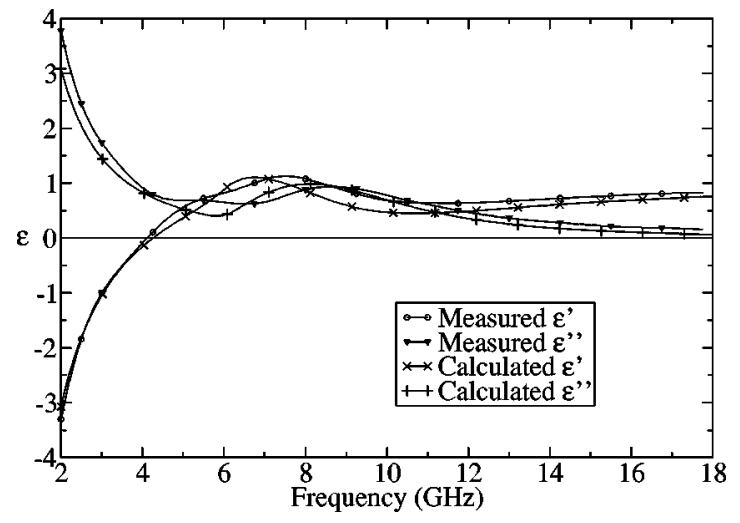

FIG. 14. Comparison between measured and calculated permittivities in the case of W3 (positive magnetostriction coefficient).

Figs. 5 and 7 that the W2 composite has a behavior similar to the composite made of copper wires.

In the case of positive magnetostriction material, a rough evaluation of the permeability can be made by ignoring the outer shell and assuming that the core domain is oriented by a uniform axial anisotropy field $H_{a}$. The circumferential permeability can be deduced from the Landau-Gilbert equation of motion (29) and by introducing the proper demagnetizing coefficients. It is given by ${ }^{16,35}$

$$
\mu_{\theta}=1+\frac{\gamma 4 \pi M_{s}\left(\gamma 4 \pi M_{s}+\gamma H_{a}+j \alpha F\right)}{\gamma^{2}\left(4 \pi M_{s}+H_{a}\right) H_{a}-F^{2}\left(1+\alpha^{2}\right)+j \gamma \alpha F\left(4 \pi M_{s}+2 H_{a}\right)},
$$

where $4 \pi M_{s}$ is the saturation magnetization, $\alpha$ the damping parameter, $\gamma \simeq 3 \mathrm{MHz} / \mathrm{Oe}$ the gyromagnetic ratio, and $F$ the frequency. The anisotropy field mainly arises from the magnetoelastic contribution. It is expected to be significantly larger for the wire W3 than for the wire W1, since they have similar ferromagnetic core and glass cover dimension that yield similar stress levels and the magnetostriction is much larger for W3. ${ }^{35}$

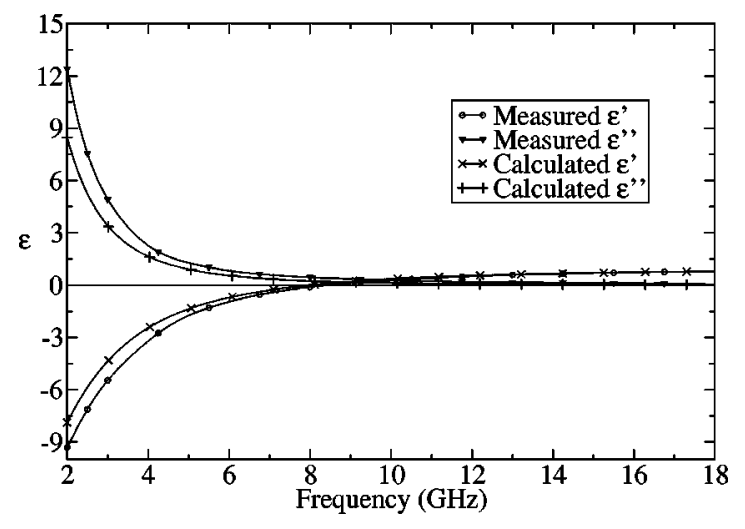

FIG. 13. Comparison between measured and calculated permittivities in the case of W2 (negative magnetostriction coefficient).
The behavior of the W3-based composite is compared in Fig. 14 to the response predicted using Eqs. (30) and (18). The computed values are obtained using the measured values of the wire radius, saturation magnetization, conductivity, and surface fraction. The best fit is obtained for $H_{a}$ $=200$ Oe and $\alpha=0.01$. The permittivity of the W1-based composite measured, Fig. 4, is compared to the prediction of the model in Fig. 15. The best fit is obtained for a $H_{a}$ smaller than for W3 composite, which is consistent with a positive magnetostriction coefficient smaller for W1 than for W3 (see Table I).

Good agreement is observed between the measurement and simulation. This indicates that the GMI approach is relevant to describe the permittivity of these composites. In particular, the resonant behavior of the permittivity observed in Figs. 3 and 4 around $4 \mathrm{GHz}$ for the W1-based composites is very well accounted for by our model. It should not be ascribed to a finite-length dipole scattering effect, though it has a similar line shape. The excellent agreement between experiment and model obtained in Figs. 13, 14, and 15 suggests that Eq. (3) is a satisfactory definition in the cases investigated here, but we should keep in mind that the presence of too large currents may limit its validity, as discussed in Ref. 26. 
TABLE II. Ferromagnetic wires parameters values: measured values and values used in Eqs. (18)-(20) and (30).

\begin{tabular}{lccccccc}
\hline \hline Parameters & $d(\mathrm{~mm})$ & $\sigma_{0}(\mathrm{~S} / \mathrm{m})$ & $f e(\mathrm{~m})$ & $\alpha$ & $H_{a}(\mathrm{Oe})$ & $4 \pi M_{s}(\mathrm{kG})$ & $a(\mu \mathrm{m})$ \\
\hline W2 measured & 10 & $7.6 e^{5}$ & $8.7 e^{-9}$ & $?$ & $?$ & $/$ & 5.25 \\
W2 model & 10 & $7.6 e^{5}$ & $8.7 e^{-9}$ & $/$ & $/$ & $/$ & 5.25 \\
W3 measured & 10 & $8 e^{5}$ & $6.4 e^{-9}$ & $?$ & $?$ & 20 & 4.5 \\
W3 model & 10 & $8 e^{5}$ & $6.4 e^{-9}$ & 0.01 & 200 & 20 & 4.5 \\
W1 measured & 5 & $7 e^{5}$ & $1.13 e^{-8}$ & $?$ & $?$ & 7.5 & 4.25 \\
W1 model & 5 & $7 e^{5}$ & $1.13 e^{-8}$ & 0.01 & 15 & 7.5 & 4.25 \\
\hline \hline
\end{tabular}

The coaxial measurments of the W1 and W2 wires (Figs. 9 and 10) are not fully consistent with the free-space measurements performed with the same wires (Figs. 3 and 5). These discrepancies may be attributed to the differences in the composite sample geometry; though in both cases the wires are excited by a parallel microwave $\mathbf{E}$ field, in the second case, infinitely vertical short dipoles sequence are considered. They can also be attributed to a difference in the magnetic domain structure due to the stress applied by the inner and outer conductors on the wire and to edge effects. ${ }^{36}$ Indeed, it has been shown that the magnetic domain structure is affected over several $\mathrm{mm}$ near the edges of microwires. ${ }^{37}$ These experimental results give evidence that the dielectric response of these composites is controlled by the magnetic properties of the wires.

The evolution of the permittivity with the bias magnetic field in the coaxial line experiments can be explained qualitatively within our framework. The results in Fig. 9 obtained with wire $\mathrm{W} 1$ are consistent with an axially magnetized wire. The bias field adds to $H_{a}$ in Eq. (30) and increases the resonance frequency, and therefore shifts the $\varepsilon^{\prime \prime}$ peak to higher frequencies. In the case of Fig. 10, the magnetization is expected to change from mainly circumferential magnetization with $\mu_{\theta}$ close to $1-j 0$ to an axial magnetization with large $\mu_{\theta}$. This should lead to large effects and, indeed, large changes in $\varepsilon$ that are actually observed for moderate bias fields. However, the complexity of the domain structure of that wire, due to the edge effects and the stress effects due to the sample holder, and also the capacitive effects due to an

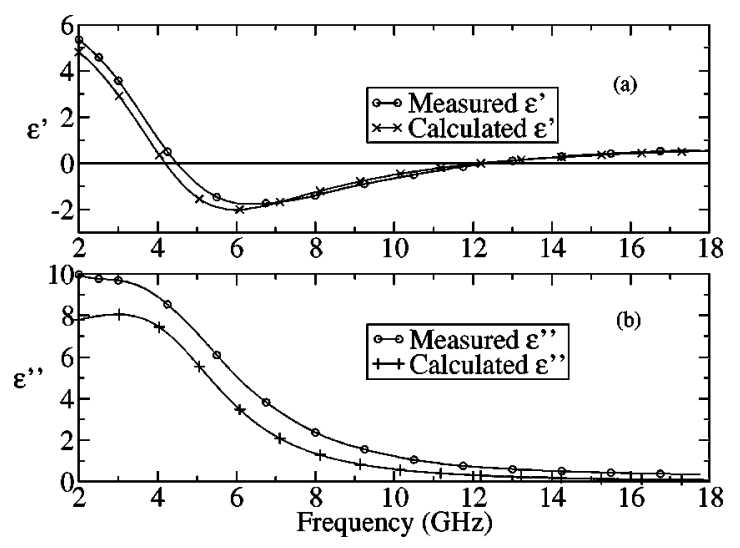

FIG. 15. Comparison between measured and calculated permittivities in the case of $\mathrm{W} 1$ (positive magnetostriction coefficient): (a) real part, (b) imaginary part. imperfect contact between the coaxial line conductors and the wire due to the glass shell, make it worthless to attempt a simulation from a simple model such as Eq. (30).

The understanding of diluted magnetic composites with elongated inclusions is sufficient to predict that it is possible to make such composites from wires, but also from thin ferromagnetic ribbons. For this configuration, the GMI approach is also quite developed ${ }^{38}$ and should be useful to design such composites. In the same way, the influence of a stress exerted on the magnetic inclusions is well known on wires and ribbons. ${ }^{39}$ For that reason, our approach should also allow the design of composites with a permittivity tuned by an external stress. As the volume fraction of magnetic inclusions is very small, significant values of stress and of adjustability should be achieved with very limited forces. The results presented here may open new opportunities to make new composite materials with engineered and tunable dielectric responses. They also open opportunities for experimental investigation of the GMI effect.

The dielectric response obtained with positive magnetostriction wires exhibits resonance peaks that are not observed in continuous nonmagnetic wires. Resonant features have been reported in composites made of nonmagnetic conductive fibers of finite length ${ }^{9}$ or frequency-selective screens. ${ }^{23}$ They are generally associated with antenna effects: a conductive strip resonates when its electrical lengh is a multiple of $\lambda / 2$. Possible diffusion or diffraction effects may be associated with this behavior. In the present investigation, the resonance is not associated with an electrical length or a percolation threshold, but with the intrinsic magnetic properties of the wires. This leads to material that may be much easier to manufacture in a reproducible way.

\section{CONCLUSION}

In this paper, the properties of composites made of diluted ferromagnetic wires have been investigated both experimentally and theoretically. It has been shown that the dielectric response is extremely dependent on the magnetic properties of the wires. It had been previously established ${ }^{9,10}$ that the permittivity of highly diluted wire-based materials is very dependent on the skin effect, in the case of nonmagnetic wires. Our results establish that the control of the skin effect through the circumferential permeability of the wires is a powerful way to engineer the dielectric response of this kind of material. It is also possible to control the permittivity of the composite through the application of an external mag- 
netic field. We derived the expressions of the effective permittivity of this type of composite starting from our definition of the permittivity. Two regimes can be defined: in the high-frequency regime, the permittivity of the wire-based composite depends only on the topology of the composite. This agrees with results already established for composites made of nonmagnetic wires, and it further demonstrates that this holds also for magnetic wires. Below this high- frequency regime, we have established that the permittivity is affected by the microwave circumferential permeability of the wire and that the underlying physics is basically the same as GMI. As the optimization of the GMI effect for a variety of magnetic wires and ribbons has been reported, this may lead to a rapid development of tunable negative index materials using arrays of magnetic wires or thin ribbons with a large GMI effect.
*Electronic address: acher@ripault.cea.fr

${ }^{1}$ D. Jaggard, A. Mickelson, and C. Papas, Appl. Phys. 18, 211 (1978)

${ }^{2}$ E. Yablonovitch, T. Gmitter, and K. Leung, Phys. Rev. Lett. 67, 2295 (1991).

${ }^{3}$ J. Pendry, Phys. World 14, 47 (2001).

${ }^{4}$ D. Smith, W. Padilla, D. Vier, S. Nemat-Nasser, and S. Schultz, Phys. Rev. Lett. 84, 4184 (2000).

${ }^{5}$ J. Pendry, Phys. Rev. Lett. 85, 3966 (2000).

${ }^{6}$ M. Wiltshire, Science 292, 60 (2001).

${ }^{7}$ J. Pendry, A. Holden, D. Robbins, and W. Stewart, IEEE Trans. Microwave Theory Tech. 47, 2075 (1999).

${ }^{8}$ A. Lagarkov and V. Kisel, Dokl. Phys. 46, 163 (2001).

${ }^{9}$ A.N. Lagarkov, S.M. Matytsin, K.N. Rozanov, and A.K. Sarychev, J. Appl. Phys. 84, 3806 (1998).

${ }^{10}$ A. Lagarkov and A. Sarychev, Phys. Rev. B 53, 6318 (1996).

${ }^{11}$ O. Acher, A. Adenot, F. Lubrano, and F. Duverger, J. Appl. Phys. 85, 4639 (2000).

${ }^{12}$ S. Prosvirnin and S. Zouhdi, AEU 55, 260 (2001).

${ }^{13}$ O. Acher, P.L. Gourriérec, G. Perrin, P. Baclet, and O. Roblin, IEEE Trans. Microwave Theory Tech. 44, 674 (1996).

${ }^{14}$ A.L. Adenot, O. Acher, T. Taffary, P. Quéffélec, and G. Tanné, J. Appl. Phys. 87, 6914 (2000).

${ }^{15}$ M. Vasquez, M. Knobel, M.L. Sanchez, R. Valenzuela, and A.P. Zhukov, Sens. Actuators A 59, 20 (1997).

${ }^{16}$ D. Makhnovskiy, L. Panina, and D. Mapps, Phys. Rev. B 63, 144424 (2001).

${ }^{17}$ S. Deprot, A.L. Adenot, F. Bertin, and O. Acher, J. Magn. Magn. Mater. 242, 247 (2002).

${ }^{18}$ A. Zhukov, J. Gonzalez, J. Blanco, M. Vazquez, and V. Larin, J. Mater. Res. 15, 2107 (2000).

${ }^{19}$ P.T. Squire, D. Atkinson, M.R.J. Gibbs, and S. Atalay, J. Magn. Magn. Mater. 132, 10 (1994).

${ }^{20}$ V. Saavedra and O. Acher (unpublished).
${ }^{21}$ J. Pendry, A. Holden, D. Robbins, and W. Stewart, J. Phys.: Condens. Matter 10, 4785 (1998).

${ }^{22}$ J. Wait, Appl. Sci. Res. 4, 393 (1955).

${ }^{23}$ N. Marcuvitz, Waveguide Handbook, IEE Electromagnetic Waves Series No. 21 (Peter Peregrinus Ltd, London, 1951).

${ }^{24}$ D. Makhnovskiy, L. Panina, D. Mapps, and A. Sarychev, Phys. Rev. B 64, 134205 (2001).

${ }^{25}$ S. Rytov, Sov. Phys. JETP 2, 466 (1956).

${ }^{26}$ O. Acher, A. Adenot, and F. Duverger, Phys. Rev. B 62, 13748 (2000).

${ }^{27}$ A. Sarychev and V. Shalaev, Phys. Rep. 335, 275 (2000).

${ }^{28}$ D. Ménard, M. Brittel, P. Ciureanu, A. Yelon, V.P. Paramonov, A.S. Antonov, P. Rudkowski, and J.O. Stroem-Olsen, J. Appl. Phys. 81, 4032 (1997).

${ }^{29}$ A. Yelon, D. Ménard, M. Britel, and P. Ciureanu, Appl. Phys. Lett. 69, 3084 (1996).

${ }^{30}$ O. Acher, P.M. Jacquart, and C. Boscher, IEEE Trans. Magn. 30, 4542 (1994)

${ }^{31}$ S. Deprot, A. Adenot, F. Bertin, and O. Acher, IEEE Trans. Magn. 37, 2404 (2001).

${ }^{32}$ D.-X. Chen, L. Pascual, F. Castano, M. Vasquez, and A. Hernando, IEEE Trans. Magn. 37, 994 (2001).

${ }^{33}$ G. Srinivasan and A. Slavin, High Frequency Processes in Magnetic Materials (World Scientific, London, 1995).

${ }^{34}$ L. Landau and E. Lifshitz, Phys. Z. Sowjetunion 8, 153 (1935).

${ }^{35}$ N. Usov, A. Antonov, and A. Largarkov, J. Magn. Magn. Mater. 185, 159 (1998).

${ }^{36}$ A. Zhukov, M. Vasquez, J. Velasquez, H. Chiriac, and V. Larin, J. Magn. Magn. Mater. 151, 132 (1995).

${ }^{37}$ M. Vasquez, C. Gomez-Polo, and D. Chen, IEEE Trans. Magn. 28, 3147 (1992).

${ }^{38}$ K. Pirota, L. Kraus, M. Knobel, P. Pagliuso, and C. Rettori, Phys. Rev. B 60, 6685 (1999).

${ }^{39}$ O. Acher, J.L. Vermeulen, P. Balcet, J. Kazandjoglou, and J. Peuzin, J. Appl. Phys. 73, 6162 (1993). 